\title{
Whole-body magnetic resonance imaging in pediatric oncology - recommendations by the Oncology Task Force of the ESPR
}

\author{
Jürgen F. Schäfer ${ }^{1}$. Claudio Granata ${ }^{2} \cdot$ Thekla von Kalle $^{3} \cdot$ Martin Kyncl $^{4} \cdot$ Annemieke S. Littooij $^{5} \cdot$ Pier Luigi Di Paolo $^{6}$. \\ Irmina Sefic Pasic ${ }^{7} \cdot$ Rutger A. J. Nievelstein ${ }^{5}$ on behalf of the Oncology Task Force of the ESPR
}

Received: 3 March 2020 / Revised: 3 March 2020 / Accepted: 16 April 2020 / Published online: 28 May 2020

(C) The Author(s) 2020

\begin{abstract}
The purpose of this recommendation of the Oncology Task Force of the European Society of Paediatric Radiology (ESPR) is to indicate reasonable applications of whole-body MRI in children with cancer and to address useful protocols to optimize workflow and diagnostic performance. Whole-body MRI as a radiation-free modality has been increasingly performed over the last two decades, and newer applications, as in screening of children with germ-line mutation cancer-related gene defects, are now widely accepted. We aim to provide a comprehensive outline of the diagnostic value for use in daily practice. Based on the results of our task force session in 2018 and the revision in 2019 during the ESPR meeting, we summarized our group's experiences in whole-body MRI. The lack of large evidence by clinical studies is challenging when focusing on a balanced view regarding the impact of whole-body MRI in pediatric oncology. Therefore, the final version of this recommendation was supported by the members of Oncology Task Force.
\end{abstract}

Keywords Cancer predisposition syndromes $\cdot$ Children $\cdot$ Magnetic resonance imaging $\cdot$ Neoplasia $\cdot$ Staging $\cdot$ Surveillance $\cdot$ Whole-body imaging

Jürgen F. Schäfer

juergen.schaefer@med.uni-tuebingen.de

1 Division of Pediatric Radiology, Department of Radiology, University Hospital of Tübingen, Hoppe-Seyler-Straße 3, 72076 Tübingen, Germany

2 Department of Paediatric Radiology, IRCCS materno infantile Burlo Garofolo, Trieste, Italy

3 Department of Pediatric Radiology, Olgahospital Klinikum Stuttgart, Stuttgart, Germany

4 Department of Pediatric Radiology, Charles University and Motol University Hospital, Prague, Czech Republic

5 Department of Radiology \& Nuclear Medicine, Princess Maxima Center for Pediatric Oncology, Wilhelmina Children's Hospital/University Medical Center Utrecht, Utrecht, The Netherlands

6 Radiology Department, Bambino Gesù Children's Hospital, Rome, Italy

7 Radiology Clinic, Sarajevo School of Science and Technology, Clinical Center University of Sarajevo,

Sarajevo, Bosnia and Herzegovina

\section{Introduction}

Whole-body MRI has been increasingly used in diagnosing pediatric oncological and non-oncological diseases over the last two decades [1-13]. Whole-body MRI is defined as a radiation-free image modality that allows a comprehensive investigation with a maximal scan length from head to toe, or minimal from base of skull to upper thigh, within a reasonable time frame. It comprises the simultaneous imaging of bone, bone marrow, soft tissue and the central nervous system. There are two ways of using this method: on the one hand, whole-body MRI is performed as a screening tool in addition to the standard imaging, mostly in coronal plane with short tau inversion recovery (STIR) sequences alone or in combination with T1-weighted images $[1,14,15]$. The advantage of short examination time has to be balanced with lower diagnostic performance in comparison to local MRI examinations. On the other hand, using both modern scanners as well as advanced sequences, the spatial resolution and the specific MRI contrasts are similar to those in local examination settings, with only some limitation regarding image quality of the extremities [13, 16-18]. In addition, the possibility of 
combining local and whole-body imaging represents a distinct advantage of the method, also allowing functional imaging (e.g., diffusion or perfusion methods) [9, 19-23]. However, to keep the examination time in an acceptable range, particularly regarding patient comfort and safety, intelligent and dedicated sequence protocols are recommended. Moreover, the high sensitivity of MRI regarding bone marrow changes has to be considered to avoid overinterpretation of normal findings in children [24]. Therefore, for specific applications and diagnostic confidence, an optimized agreement of sequence numbers, spatial resolution and acquisition time must be realized. This issue is unquestionably challenging and needs further harmonization.

Regarding local staging and response assessment of solid malignant tumors in children and adolescents, MRI is the modality of choice in most cases, whereas whole-body MRI represents a non-standard method and is not generally considered in the protocols of the international oncological pediatric societies (e.g., International Society of Paediatric Oncology, or SIOP). However, in contrast, at a number of institutions whole-body MRI is now integrated into the diagnostic workup in children with cancer. Unfortunately, the diagnostic value has been evaluated mostly in small single-center projects. It is primarily in this context that the value of whole-body MRI for detecting osteomedullary metastases has been assessed [1, 3, 15, 25, 26]. But tumor spread into the abdominal organs, lymph nodes and the central nervous system has also been increasingly investigated with whole-body MRI [2, 6, 7, 27-29], with increasing accuracy when diffusion-weighted sequences are added to the imaging protocol $[9,30]$. Most trials have compared the diagnostic accuracy of whole-body MRI with conventionally used imaging workups including metabolic imaging. Only a few studies have measured the predictive value of whole-body MRI findings or the change in patient outcome [5]. A relatively new application of wholebody MRI is its use in cancer predisposition disorders as a significant screening procedure, which is now established for some diseases, with an increasing database (e.g., LiFraumeni syndrome) [11, 31-38].

In summary, on the one hand, whole-body MRI exists as a valuable tool in the daily practice of pediatric oncology and is frequently performed in specialized centers. On the other hand, there are major concerns about the level of scientific evidence. Hence, the presented recommendations summarize a consensus statement by experts in the field of whole-body MRI where large evidence is lacking. Finally, the confusion about the protocols and techniques needs to be harmonized. Therefore, this recommendation of the Oncology Task Force aims to specify applications of whole-body MRI, to describe the technical pre-requisition, and to define useful protocols to maximize the diagnostic accuracy and confidence.

\section{Key recommendations}

- Whole-body MRI enables the investigation of systemic spread of solid malignant tumors.

- Whole-body MRI is useful for surveillance in cancer predisposition syndromes.

- Imaging protocol adaption to each child's age and the indication is mandatory to maximize diagnostic performance.

- The high sensitivity of STIR MRI regarding bone marrow changes has to be considered to avoid overinterpretation of normal findings.

- Additional imaging might be necessary in particular tumor entities or diagnostic scenarios.

\section{Specific tumors}

\section{Lymphoma}

Hodgkin lymphoma and non-Hodgkin lymphoma together are the third most common form of malignancy in children and adolescents [39]. Non-Hodgkin lymphoma is most frequent in children younger than 15 years, whereas Hodgkin lymphoma is predominantly diagnosed in teenagers. Pediatric lymphomas are staged using the Lugano classification for Hodgkin lymphoma [40] and the International Pediatric Non-Hodgkin Lymphoma Staging System for non-Hodgkin lymphoma [41].

Standard imaging procedure: In Hodgkin lymphoma, [F-18]2-fluoro-2-deoxyglucose (FDG) positron emission tomography (PET)/CT remains the first-line modality for staging and response assessment, providing both structural and functional metabolic information [40]. In non-Hodgkin lymphoma the current guidelines propose to perform chest radiograph, ultrasonography (US) of the abdomen and cranial/ spinal MRI if indicated, except for the B cell non-Hodgkin lymphoma, where FDG PET or whole-body MRI is recommended.

Review and comments on the literature: Because both PET and contrast-enhanced CT involve substantial radiation exposure and children often undergo several PET/CT examinations during the course of treatment, there is an increasing interest in the use of whole-body MRI as a good radiation-free alternative. Several studies have shown that whole-body MRI is feasible even in children [2, 8, 29, 42]. Table 1 shows proposed whole-body MRI sequences for use in children with lymphoma.

Staging: In a study by Punwani et al. [29], the authors reported very good agreement for nodal and extranodal disease involvement between whole-body MRI compared to an FDG PET/CT reference standard, despite only using STIR for wholebody MRI. Because of the clear visualization of lymphoid 
Table 1 Proposed magnetic resonance imaging protocol at $1.5 \mathrm{~T}$ in lymphoma (please also refer to Technical Considerations)

\begin{tabular}{|c|c|c|c|c|c|}
\hline Sequence & T1-W TSE & T2-W STIR & DWI & T2-W STIR/SPAIR ${ }^{a}$ & ceT1-W FS ${ }^{\mathrm{a}}$ \\
\hline Orientation & Coronal & Coronal & Axial & Axial & Axial \\
\hline $\begin{array}{l}\text { Respiratory motion } \\
\text { compensation }\end{array}$ & $\begin{array}{l}\text { Breath hold (thorax } \\
\text { and abdomen) }\end{array}$ & $\begin{array}{l}\text { Respiratory triggering } \\
\text { (thorax and abdomen) }\end{array}$ & Free breathing & $\begin{array}{l}\text { Respiratory triggering } \\
\text { (thorax and abdomen) }\end{array}$ & Breath hold ${ }^{\mathrm{b}}$ \\
\hline Anatomical coverage & Head to groin & Head to groin & Head to groin & Head to groin & Head to groin \\
\hline
\end{tabular}

ceT1-W contrast-enhanced T1-weighted, DWI diffusion-weighted imaging, FS fat saturation, SPAIR spectrally adiabatic inversion recovery, STIR short tau inversion recovery, $T 1-W$ T1-weighted, $T 2-W$ T2-weighted, TSE turbo spin echo

${ }^{\text {a }}$ Either axial T2-W STIR/SPAIR or ceT1-W FS, optional

${ }^{\mathrm{b}}$ Free breathing with radial imaging

tissue, diffusion-weighted imaging (DWI) is a potentially interesting additional sequence to use for evaluating lymphoma. Regacini et al. [30] showed an excellent sensitivity for staging pediatric lymphoma compared to contrast-enhanced CT using coronal STIR and DWI sequences. However, other studies could not demonstrate the additional value of DWI to conventional MRI sequences in staging pediatric lymphoma $[6,8]$. This could be related to the fact that both benign and malignant nodes demonstrate impeded diffusion. Therefore, the detection of lymph nodes in whole-body DWI is still mainly based on size criteria. Overall, in pediatric Hodgkin lymphoma, wholebody MRI with DWI agreed with an FDG PET/CT-based reference for disease stage in $82-85 \%$ of cases [8, 42]. Interestingly, a study performed by Latifoltojar et al. [42] used a highest $b$ value of $500 \mathrm{~s} / \mathrm{mm}^{2}$. The authors acknowledged that using higher $\mathrm{b}$ values could decrease perceptual errors for extranodal disease assessment. Indeed, higher $\mathrm{b}$ values are preferred to optimize background body signal suppression and improve lesion conspicuity, in particular in organs/regions with normal intrinsic diffusion restriction.

Response assessment: Early recognition of therapy response or failure to chemotherapy enables better selection of children who need more or less intensive therapy. The concept of early response assessment with FDG PET/CT in lymphoma has received considerable attention in the last few years, although it is still not officially recommended outside clinical trials [40]. The role of whole-body MRI in response assessment in pediatric lymphoma is still under investigation. Mayerhoefer et al. [43] showed in 64 adults with lymphoma that whole-body MRI with DWI could serve as a feasible alternative for FDG PET/CT during follow-up and treatment response assessment. Several, mostly pilot, studies compared the quantitative data from FDG PET/CT (standardized uptake value [SUV]) with DWI (apparent diffusion coefficient $[A D C]$ values) for early response assessment, with inconclusive results. They reported presence or absence of an inverse correlation between ADC and SUV [22, 23, 29, 44]. Latifoltojar et al. [42] recently published their results of a prospective study in 55 children with Hodgkin lymphoma and showed that whole-body MRI was correct in $66 \%$ and underestimated response in $26 \%$ of cases during interim response assessment.

According to the currently available literature, whole-body MRI is a good alternative for contrast-enhanced $\mathrm{CT}$ in staging pediatric lymphoma. However, FDG PET with low-dose CT for attenuation correction remains vital for interim response assessment and therefore cannot be omitted during staging or interim response assessment in lymphoma.

Key recommendation in pediatric lymphoma: Wholebody MRI is a good alternative for contrast-enhanced CT in staging pediatric lymphoma. However, for FDG-avid lymphomas, 18F-FDG PET remains vital for staging and response assessment.

\section{Neuroblastoma}

Neuroblastoma is a neoplasm arising from primordial neural crest cells and is the second most common solid extracranial tumor in children, accounting for about $6 \%$ of all childhood cancers [45]. It can occur anywhere along the sympathetic chain from neck to pelvis, with the adrenal medulla as the most common site at presentation. In about $70 \%$ of cases, metastatic disease is observed at diagnosis, including the liver, lymph nodes, bone marrow, cortical bone and skin. About $90 \%$ of cases are diagnosed before the age of 6 years. The outcome is variable because neuroblastoma can spontaneously regress in children younger than 1 year, whereas in older children it can cause death despite aggressive treatment with surgery, chemotherapy, bone marrow transplantation and radiotherapy [46].

Standard imaging procedure: Accurate staging is pivotal for planning treatment. Furthermore, a systematic assessment of the relationship between the primary tumor and the adjacent structures according to a series of imaging-defined risk factors is mandatory to evaluate tumor resectability [47]. Typically, CT or MRI, iodine-123 metaiodobenzylguanidine (I-123 MIBG) scintigraphy and bone marrow biopsies are used to evaluate local and metastatic disease at diagnosis and during treatment. Further imaging modalities (e.g., FDG PET in 
MIBG-negative tumors) and frequency of imaging in followup are also related to risk groups.

Review and comments on the literature: The availability of studies on the role of whole-body MRI in neuroblastoma is still very limited. Furthermore, some of them included children not only with neuroblastoma, but also with other common pediatric tumors. In 2003, Pfluger et al. [48] retrospectively studied 28 people with neuroblastoma who had 50 I-123 MIBG scans in combination with 50 MRI studies. MRI studies included fast spin-echo T1-weighted and T2weighted images, STIR images and contrast-enhanced T1weighted images of suspected lesions. They concluded that MRI showed a higher sensitivity and I-123 MIBG a higher specificity, but that integrated imaging with both I-123 MIBG and MRI allowed an increase in both sensitivity and specificity.

In 2013, Siegel et al. [7] compared whole-body MRI with conventional imaging for detecting distant metastases in children with common malignant tumors. Sixty-six children with newly diagnosed lymphoma, neuroblastoma or soft-tissue sarcoma were selected for image review and analysis [7]. The authors concluded that the non-inferior accuracy for diagnosing distant metastases (e.g., pulmonary metastases) was not established for the use of whole-body MRI compared with conventional methods. However, improved accuracy was seen with whole-body MRI in children with nonlymphomatous tumors [7]. In 2014, Kembhavi et al. [49] assessed the diagnostic accuracy of whole-body MRI for metastatic disease in people with small round cell tumors including neuroblastoma, primitive neuroectodermal tumor and rhabdomyosarcoma by comparison with routine staging procedures. Whole-body MRI studies included coronal T1-W and STIR sequences. They concluded that whole-body MRI had high diagnostic accuracy for evaluating metastatic disease to the marrow [49]. On the contrary, the detection rate of nodal metastases was less when whole-body MRI was compared with conventional staging, and chest CT was still essential for accurate evaluation of lung metastases.

Diffusion-weighted imaging can be effectively integrated in MRI studies in children with neuroblastic tumors: Peschmann et al. [50] observed in 19 people that ADC values at diagnosis differed significantly between malignant and benign neuroblastic tumors. Furthermore, low baseline ADC was predictive of tumor progression and relapse. With therapy, increasing ADC appeared to predict relapse-free survival, whereas a decreasing ADC during therapy was an indicator of poor prognosis [50]. Very recently, Ishiguchi et al. [19] studied the role of whole-body DWI with background body suppression using only singular high $b$ value imaging in detection of lymph node and bone metastases from neuroblastoma. Thirteen people underwent both 18F-FDG PET/CT and whole-body DWI with background body suppression. According to the results of this study, whole-body DWI with background body suppression showed a similar level of sensitivity for detecting lymph node metastases to that of FDG PET/CT [19]. However, without ADC mapping, the specificity was poor in this study [19].

The studies on the role of whole-body MRI in neuroblastoma are still scarce, based on a limited number of patients, and in some cases with contradictory results. Nevertheless, whole-body MRI could be a promising, radiation-free modality for detecting bone and bone marrow metastases (Table 2 indicates proposed sequences). Furthermore, the ADC value based on DWI of the primary tumor could be a good indicator of outcome. However, there is still a strong need for prospective large cohort studies to validate the role of whole-body MRI in children with neuroblastoma.

Key recommendations in neuroblastoma: Whole-body MRI could play a role as an ancillary study for detecting bone and bone marrow disease, while performing MRI for evaluation of the primary tumor. Prospective multicenter studies are needed to validate the role of whole-body MRI versus the current reference standards in pediatric neuroblastoma.

\section{Sarcomas}

Pediatric sarcomas are a heterogeneous group of rare tumors, most of which are highly malignant. They account for approximately $10-15 \%$ of solid malignancies in childhood and adolescence. Soft-tissue sarcomas are divided into two groups: rhabdomyosarcomas and non-rhabdomyosarcoma soft-tissue sarcomas. The most common primary malignant bone tumors are osteosarcomas and Ewing sarcomas.

Standard imaging procedure: A multidisciplinary diagnostic and therapeutic approach is mandatory in all cases and should be carried out by a reference center [51, 52]. In softtissue sarcomas, conventional imaging and staging as recommended by international study groups consist of highresolution MRI for local disease and loco-regional lymph nodes, CT for lung metastases, and bone scintigraphy for skeletal metastases. Imaging with FDG PET/CT is optional $[51,52]$ - depending on local availability (European Paediatric Soft-tissue Sarcoma Study Group rhabdomyosarcoma [EpSSG RMS2005] protocol, German Cooperative Weichteilsarkom Studiengruppe [CWS] guidance 2012) or is recommended at baseline and in cases of suspected tumor recurrence (Children's Oncology Group Soft Tissue Sarcoma Committee). Bone sarcomas additionally require radiographs of the primary tumor and the search for skip lesions in the same extremity by MRI.

Review and comments on the literature: Apart from bone scintigraphy, whole-body imaging was not generally recommended [51, 52] until the most recent EpSSG guideline, which recommends performing FDG PET/CT (MR) for staging at baseline and after three courses of chemotherapy. Its 
Table 2 Proposed magnetic resonance imaging protocol in neuroblastoma (please also refer to Technical Considerations)

\begin{tabular}{|c|c|c|c|c|c|}
\hline Sequence & T2-W STIR & T2-W STIR ${ }^{\mathbf{a}}$ & DWI & T2-W STIR/SPAIR & ceT1-W FS \\
\hline Orientation & Coronal & Sagittal & Axial & Axial & Axial \\
\hline $\begin{array}{l}\text { Respiratory motion } \\
\text { compensation }\end{array}$ & $\begin{array}{l}\text { Respiratory triggering } \\
\text { (thorax and abdomen) }\end{array}$ & Free breathing & Free breathing & $\begin{array}{l}\text { Respiratory triggering } \\
\text { (thorax and abdomen) }\end{array}$ & Breath hold ${ }^{\mathrm{b}}$ \\
\hline Anatomical coverage & Whole-body & Spine & $\begin{array}{l}\text { Whole-body or } \\
\text { affected regions }\end{array}$ & Head to groin & Head to groin \\
\hline
\end{tabular}

ceT1-W contrast-enhanced T1-weighted, DWI diffusion-weighted imaging, FS fat saturation, SPAIR spectrally adiabatic inversion recovery, STIR short tau inversion recovery, $T 1-W$ T1-weighted, T2-W T2-weighted, TSE turbo spin echo

${ }^{\text {a } O p t i o n a l ~}$

${ }^{\mathrm{b}}$ Free breathing with radial imaging

application is especially important in suspected disseminated disease because a curative therapeutic approach requires the local control of each single lesion $[52,53]$. The complete depiction of the whole body from head to toe is mandatory, be it to detect all skeletal metastases [26], distant metastases in unexpected localizations [54], or a small primary of a disseminated alveolar rhabdomyosarcoma in a hand or foot [55] (Table 3).

Given the young patient age and the high number of follow-up examinations, a modality without exposure to ionizing radiation such as whole-body MRI would be preferable. Whole-body MRI allows for excellent contrast resolution of soft tissue and bone marrow. It provides versatile options such as DWI and contrast enhancement. With a thoroughly planned imaging protocol and a thoughtful choice of MR coils, one examination might simultaneously give an overview of the tumor spread as well as high-resolution images of a tumor site.

Although most experts employ coronal STIR sequences, there is, however, neither a universally accepted standard protocol nor a clearly defined set of assessment criteria. Comparative evaluation of whole-body MRI is therefore difficult. Several studies found that for sarcomas and other solid tumors, whole-body imaging conducted as PET/CT or wholebody MRI outperforms conventional imaging in the detection of metastases $[1,7,15,54,56]$. Other results are, however, inconsistent. In older studies, whole-body MRI has been found to be equally sensitive [27] as well as less sensitive [1] as compared to FDG PET. It is also known to detect fewer lung metastases than conventional imaging [7]. PET/CT has, because of its metabolic information, higher sensitivity for detecting nodal disease [7]. An early study showing the possible impact of whole-body MRI on patient outcome was limited by small numbers [5]. A meta-analysis on the detection of skeletal metastases in children with primary solid tumors found data to be too scarce and heterogeneous to recommend whole-body MRI as an alternative. However, $3 / 5$ of these studies included PET as a reference standard, which is also not generally recommended [26]. Qualified studies on the role

Table 3 Proposed magnetic resonance imaging protocol in sarcoma (please also refer to Technical Considerations)

\begin{tabular}{|c|c|c|c|c|c|c|}
\hline MR sequence & T2-W STIR/SPAIR & T2-W STIR/SPAIR & DWI & ceT1-W FS & $\begin{array}{l}\text { T2-W STIR } \\
\text { HR }^{\mathrm{a}}\end{array}$ & $\begin{array}{l}\text { T1-W HR before and } \\
\text { with FS after contrast }{ }^{\mathrm{a}}\end{array}$ \\
\hline Orientation & Coronal & Axial & Axial & Axial & $\begin{array}{l}2 \text { planes, } \\
\text { depending on } \\
\text { tumor site }\end{array}$ & Multiple planes ${ }^{\mathrm{b}}$ \\
\hline $\begin{array}{l}\text { Respiratory } \\
\text { motion } \\
\text { compensation }\end{array}$ & $\begin{array}{l}\text { Respiratory triggering } \\
\text { (thorax and } \\
\text { abdomen) }\end{array}$ & $\begin{array}{l}\text { Respiratory triggering } \\
\text { (thorax and } \\
\text { abdomen) }\end{array}$ & Free breathing & Breath hold ${ }^{\mathrm{a}}$ & Free breathing & Free breathing \\
\hline $\begin{array}{c}\text { Anatomical } \\
\text { coverage }\end{array}$ & Whole body ${ }^{c}$ & Head to groin & $\begin{array}{l}\text { Whole-body or } \\
\text { affected } \\
\text { regions }\end{array}$ & Head to groin & $\begin{array}{l}\text { Primary tumor } \\
\text { with dedicated } \\
\text { coil }\end{array}$ & $\begin{array}{l}\text { Primary tumor with } \\
\text { dedicated coil }\end{array}$ \\
\hline
\end{tabular}

ceT1-W contrast-enhanced T1-weighted, $D W I$ diffusion-weighted imaging, $F S$ fat saturation, $H R$ high resolution, SPAIR spectrally adiabatic inversion recovery, STIR short tau inversion recovery, $T 1-W$ T1-weighted, $T 2-W$ T2-weighted

${ }^{a}$ Optional for dedicated imaging of primary tumor site and single metastases; please refer to EpSSG rhabdomyosarcoma imaging guideline for further recommendations

${ }^{\mathrm{b}}$ At least two planes after contrast administration

${ }^{\mathrm{c}}$ Especially in alveolar rhabdomyosarcoma, extremities should be completely depicted [55] 
of diffusion-weighted imaging are also rare, especially in children with rhabdomyosarcoma [57]. DWI might be helpful in staging and therapy monitoring, although diffusion restriction is not specific for malignant lesions and is also typical of normal lymphatic tissue. In osteosarcoma, DWI helps to predict tumor response to neo-adjuvant therapy [58], but larger prospective studies need to be performed.

It seems reasonable that in highly malignant sarcomas the most effective search for metastases and relapses should be preferred even if associated with radiation exposure. However, bone scintigraphy is the only whole-body modality that is mandatory in basic imaging, whereas FDG PET/CT is only optional. To detect relapse, the use of cross-sectional imaging has not been demonstrated to be more beneficial or cost-effective than clinical assessment and chest radiographs alone. Thus, prospective clinical studies are needed, first to define patients who would benefit from whole-body imaging, and second to identify the most effective of the available modalities (whole-body MRI, PET/CT or PET/MRI). Wholebody MRI might, at present, be considered in addition to basic imaging recommendations for children who would probably benefit from whole-body imaging without increasing their cumulative radiation dose.

Key recommendations in sarcomas: Whole-body MRI could be considered in addition to imaging recommendations of international oncology groups. Children with sarcoma who have disseminated disease might benefit from whole-body MRI without increasing their cumulative radiation dose.

\section{Langerhans cell histiocytosis}

Langerhans cell histiocytosis is characterized by accumulation of clonal CD1a-positive immature dendritic cells, so-called Langerhans cell histiocytosis cells, together with eosinophils, macrophages, lymphocytes and osteoclast-like giant cells. In children younger than 15 years, incidence of Langerhans cell histiocytosis is $4-5$ cases per million per year [59]. Langerhans cell histiocytosis can affect many organs, including the skeleton, skin, lymph nodes, liver, lungs, spleen, hematopoiesis and central nervous system. Previously, Langerhans cell histiocytosis included three entities: eosinophilic granuloma, Hand-Schüller-Christian disease and Letterer-Siwe disease, but at present it is classified as unifocal, single-system multifocal, or multifocal multisystem disease [59]. Children with single-system or organ Langerhans cell histiocytosis, such as skeleton, skin or lymph nodes, have an excellent prognosis and need minimal or sometimes no treatment at all. The outcome of children with multisystem Langerhans cell histiocytosis can range from spontaneous resolution to fatal outcome despite treatment. Therefore, it is of the utmost importance to stratify these patients according to unifocal versus multifocal disease.
Standard imaging procedure: Diagnostic evaluation and treatment are based on the ongoing LCH-IV International Collaborative Treatment Protocol for Children and Adolescents with Langerhans cell histiocytosis (EudraCT Nr.: 2011-001699-20). According to this protocol, diagnostic imaging at onset should include an abdominal US study for evaluating size and structure of the liver and spleen, a chest radiograph and a radiologic skeletal survey. Functional imaging like bone scan or FDG PET is optional and can be performed in addition to the skeletal survey. Chest $\mathrm{CT}$ is needed in case of lung involvement, whereas head CT or MRI should be performed in case of craniofacial lesions or mastoid involvement. Neurologic abnormalities or suspected endocrine abnormalities require a head MRI, and MRI of the spine is necessary in cases of suspected vertebral lesions.

Review and comments on the literature: Langerhans cell histiocytosis can be a multisystem and multifocal disease. Therefore, whole-body MRI could be an excellent method for evaluating the whole body in one examination. Nevertheless, very few studies with preliminary results on the role of whole-body MRI in Langerhans cell histiocytosis are available. The number of children included in these studies is limited (range 2 to 46) [3, 25, 60-62]. Three of five available studies investigated the role of whole-body MRI at diagnosis for primary staging or follow-up; in two studies only the role of whole-body MRI at diagnosis was investigated $[3,25$, 60-62]. STIR sequence was performed in all studies; in one of them it was the only sequence performed [61]. T1-W fast spinecho (FSE) sequences with and without contrast enhancement were performed in two studies [60, 62], T1-W FSE without contrast enhancement in one study [25], and T1-W FSE with just contrast enhancement in another study [3]. All studies included coronal and sagittal images, whereas only one study also included axial images [62].

The standard of reference for comparison with whole-body MRI was skeletal survey or plain radiographs in four studies $[3,25,60,61]$, in combination with skeletal scintigraphy in three of them [3, 60, 61]. Histopathology or follow-up imaging was the standard of reference in another study [62]; this study compared the performances of FDG PET with wholebody MRI in Langerhans cell histiocytosis. Sensitivity and specificity of whole-body MRI for lesion detection in Langerhans cell histiocytosis ( $81 \%$ and $47 \%$, respectively) were reported only in the study by Mueller et al. [62], whereas Kim et al. [60] reported a sensitivity of $99 \%$.

According to the results of their study, Steinborn et al. [25] concluded that whole-body MRI had a higher detection rate of bony lesions than a skeletal survey, and they therefore suggested that whole-body MRI be the imaging modality of choice for assessing skeletal involvement in Langerhans cell histiocytosis. Similarly, Goo et al. [3] and Laffan et al. [61] reported that whole-body MRI can be an excellent imaging tool for assessing skeletal involvement. Furthermore, Goo 
et al. [3] concluded that whole-body MRI can also detect extraskeletal disease, and they suggested that changes in signal intensity on STIR and T1-W contrast-enhanced images could demonstrate response to treatment in active lesions. Mueller et al. [62] studied the diagnostic value of FDG PET and MRI in pediatric Langerhans cell histiocytosis. They concluded that MRI showed higher sensitivity than FDG PET in lesion detection, whereas FDG PET showed higher specificity. Interestingly, they also reported that FDG PET was more accurate than MRI in evaluating disease activity after chemotherapy because they observed persisting residual contrast enhancement and T2 hyperintensity in lesions with no residual FDG uptake on PET. Probably the most interesting results based on the largest case series so far - come from a recent study by Kim et al. [60]. They concluded that whole-body MRI had higher detectability for Langerhans cell histiocytosis lesions than skeletal survey and bone scintigraphy, with no significant differences in the number of false-positives per patient, while the three modalities had comparable accuracy in the initial staging. Table 4 presents a summary of these studies' findings. Table 5 presents a whole-body MRI protocol for Langerhans cell histiocytosis.
According to the scientific literature available, whole-body MRI could be the imaging modality of choice for assessing skeletal involvement at onset, thus replacing radiologic skeletal survey and bone scintigraphy. Conventional radiologic studies could be performed only of bones with positive findings on whole-body MRI. Whole-body MRI should include at least T1-W FSE and STIR images in coronal and sagittal planes, whereas it is unclear whether T1-W contrast-enhanced images improve the accuracy of the study. None of the published studies assessed the role of diffusion-weighted imaging. The role of whole-body MRI during follow-up seems to be questionable because persisting signal abnormalities could be caused by post-therapy tissue reorganization. In addition, the role of whole-body MRI in evaluating extraskeletal disease is promising but not finally approved. New and larger prospective multicenter studies are therefore needed.

Key recommendations in Langerhans cell histiocytosis: Whole-body MRI could replace skeletal survey and bone scintigraphy for assessing skeletal involvement at onset. Its role in assessment of extraosseous disease and followup is still unclear. Thanks to its high sensitivity and accuracy, whole-body MRI should always be considered in

Table 4 Summary of published data on whole-body magnetic resonance imaging in Langerhans cell histiocytosis

\begin{tabular}{|c|c|c|c|c|c|}
\hline & Goo et al. [3] & Laffan et al. [61] & $\begin{array}{l}\text { Steinborn et al. } \\
{[25]}\end{array}$ & Mueller et al. [62] & Kim et al. [60] \\
\hline Number of patients & 9 & 2 & 14 & 15 & 46 \\
\hline Staging at diagnosis & Yes & Yes & Yes & Yes & Yes \\
\hline Follow-up & Yes & No & Yes & Yes & No \\
\hline $\begin{array}{l}\text { Total number of } \\
\text { lesions observed }\end{array}$ & NA & NA & NA & 53 & 105 \\
\hline $\begin{array}{l}\text { Number of lesions } \\
\text { observed on } \\
\text { primary staging }\end{array}$ & NA & NA & NA & 25 & 105 \\
\hline $\begin{array}{l}\text { Number of lesions } \\
\text { observed on } \\
\text { follow-up }\end{array}$ & NA & NA & NA & 28 & NA \\
\hline Sequences & STIR, T1-W FSE CE & STIR & T1-W FSE, STIR & $\begin{array}{l}\text { T1-W FSE, STIR, T1-W FSE CE + } \\
\text { dedicated study of the brain } \\
\text { with T1-W FSE, T2-W FSE, } \\
\text { FLAIR, T1-W FSE CE }\end{array}$ & $\begin{array}{l}\text { STIR, T1-W FSE, } \\
\text { T1-W FSE CE }\end{array}$ \\
\hline Acquisition planes & $\begin{array}{l}\text { Coronal, sagittal } \\
\text { limited to the } \\
\text { trunk }\end{array}$ & Coronal and sagittal & $\begin{array}{l}\text { Coronal and } \\
\text { sagittal }\end{array}$ & Axial, coronal, sagittal & Coronal, sagittal \\
\hline $\begin{array}{c}\text { Standard of } \\
\text { reference }\end{array}$ & $\begin{array}{l}\text { RX skeletal survey, } \\
\text { bone scintigraphy }\end{array}$ & $\begin{array}{l}\text { Skeletal scintigraphy } \\
\text { and/or plain } \\
\text { radiographs }\end{array}$ & $\begin{array}{l}\text { RX skeletal } \\
\text { survey }\end{array}$ & $\begin{array}{l}\text { Histopathology and/or follow-up } \\
\text { of lesions. Whole-body MRI } \\
\text { performances were also compared } \\
\text { with } 18 \mathrm{~F}-\text {-FDG PET }\end{array}$ & $\begin{array}{l}\text { Histopathology } \\
\text { and/or follow-up } \\
\text { of lesions }\end{array}$ \\
\hline Sensitivity & NA & NA & NA & $81 \%$ & $99 \%$ \\
\hline Specificity & NA & NA & NA & $47 \%$ & NA \\
\hline
\end{tabular}

$C E$ contrast-enhanced, $D W I$ diffusion-weighted imaging, FDG PET [F-18]2-fluoro-2-deoxyglucose positron emission tomography, FLAIR fluid-attenuated inversion recovery, FS fat saturation, FSE fast spin echo, NA not available, $R X$ radiograph, SPAIR spectrally adiabatic inversion recovery, STIR short tau inversion recovery, $T 1-W$ T1-weighted, $T 2-W$ T2-weighted, TSE turbo spin echo 
Table 5 Proposed whole-body magnetic resonance imaging protocol in Langerhans cell histiocytosis (please also refer to Technical Considerations)

\begin{tabular}{|c|c|c|c|c|c|}
\hline Sequences & T1-W TSE & T2-W STIR & T2-W STIR & T2-W STIR ${ }^{a}$ & T1-W TSE ${ }^{a}$ \\
\hline Orientation & Coronal & Coronal & Sagittal & Axial & Axial \\
\hline $\begin{array}{l}\text { Respiratory motion } \\
\text { compensation }\end{array}$ & $\begin{array}{l}\text { Breath hold } \\
\text { (thorax and abdomen) }\end{array}$ & $\begin{array}{l}\text { Respiratory triggering } \\
\text { (thorax and abdomen) }\end{array}$ & Free breathing & Free breathing & Free breathing \\
\hline Anatomical coverage & Head to toe & Head to toe & Spine & Head & Head \\
\hline
\end{tabular}

STIR short tau inversion recovery, T1-W T1-weighted, T2-W T2-weighted, TSE turbo spin echo

${ }^{\text {a }}$ Optional

children with Langerhans cell histiocytosis at onset, especially if sedation is not needed.

\section{Screening in cancer predisposition disorders}

Cancer predisposition syndromes comprise entities characterized by a risk of development of specific tumors or co-occurrence of tumors caused by a germ-line mutation in one or more cancer-related genes [63]. These can manifest from infancy to adulthood and are of unknown penetrance, variable incidence, and differing age of onset. At least $10 \%$ of children with cancer harbor a diseaseassociated pathogenic variant in a known cancer predisposition gene. Common cancer predisposition syndromes in children include Li-Fraumeni syndrome, constitutional mismatch repair deficiency syndrome (CMMRD), hereditary paraganglioma, pheochromocytoma syndrome, rhabdoid tumor predisposition syndrome, hereditary retinoblastoma, and Neurofibromatosis 1. Cancers typically related to Li-Fraumeni syndrome are osteosarcoma, adrenocortical carcinoma, brain tumors, soft-tissue sarcoma and premenopausal breast cancer [37, 64]. Lower-risk sites are the bowel, bone marrow and skin. Children with hereditary retinoblastoma are at risk of developing osteosarcoma; soft-tissue sarcoma; nasal/orbital tumor; melanoma; and lung, bladder, breast and uterine carcinoma [65]. Cancers related to CMMRD are brain tumors, gastrointestinal and hematologic malignancies [31]. Identification of an underlying cancer predisposition syndrome could lead to a recommendation of health surveillance or prophylactic surgery.

Standard imaging procedure: The role of imaging in cancer predisposition syndromes is to be part of tumor surveillance and to allow for detection of tumor recurrence. Furthermore, imaging enables assessment of individuals and family members identified as being at risk for tumors on the basis of an abnormal genetic test result. Because of the increased sensitivity of children to ionizing radiation, most surveillance imaging protocols rely more on US and MRI (whole-body MRI) than nuclear medicine and CT $[10,11]$. Because the cancer spectrum in cancer predisposition syndromes is age-dependent, screening modalities and frequency change depending on the gender and age of the patient (e.g., adrenocortical cancer risk in very young children and high breast cancer risk in young woman with Li-Fraumeni syndrome; specific surveillance of the gastrointestinal tract, the central nervous system, and the hematopoietic system in infancy; and surveillance of the genitourinary tract in older children with CMMRD).

Review and comments on the literature: Variations in screening protocols arise from institutional preference and technological capabilities. Whole-body MRI can be performed with the additional use of regional MRI (e.g., dedicated brain MRI in Li-Fraumeni syndrome, CMMRD and hereditary retinoblastoma, or targeted MRI of specific organs and extremities) $[10,11]$. It provides head-to-toe coverage, displayed mostly as whole-body fused coronal images. A coronal fluid-sensitive 2-D sequence (STIR) represents the key sequence of a whole-body MRI screening protocol. T1weighted images are applied for better tissue characterization (in particular regarding bone marrow signal). Whole-body DWI, typically acquired axially, is now incorporated into whole-body pediatric protocols. However, DWI and ADC measurements at diagnosis and follow-up must still be validated [66]. Generally, whole-body MRI can be performed without general anesthesia in children with a developmental age of 6 years and older.

In a retrospective study of 50 whole-body MRIs in 24 pediatric patients with cancer predisposition syndromes, Anupindi et al. [31] demonstrated that whole-body MRI is a method with a very high negative predictive value (NPV; $100 \%$ ). Friedman et al. [38] reported an NPV of $97 \%$ in a total of 41 whole-body MRI screening tests performed in survivors of hereditary retinoblastoma. This would indicate that children with a normal whole-body MRI are unlikely to have a tumor. Villani et al. [65] reported in 2016 that long-term compliance with a comprehensive surveillance protocol in individuals with pathogenic TP53 variants is associated with improved long-term survival. Their protocol included physical examination and frequent biochemical and imaging studies (consisting of whole-body MRI, brain MRI, breast MRI, mammography, abdominal and pelvic ultrasound, and colonoscopy). O'Neill 
et al. [34] recently postulated that it is feasible to use wholebody MRI for tumor surveillance in pediatric patients with $\mathrm{Li}-$ Fraumeni syndrome in his group of 22 patients. In Neurofibromatosis 1, routine MRI surveillance is not recommended unless children are symptomatic or with an already diagnosed tumor [37].

From these studies, it can be concluded that whole-body MRI complemented with targeted imaging (organ, brain) when indicated can properly fulfill the need for a radiationfree method for early tumor detection in pediatric cancer predisposition syndromes. However, Anupindi et al. [31] showed that most people in their study (96\%) had incidental findings of no significant clinical impact. False-positive findings can be a potential risk in whole-body MRI surveillance of cancer predisposition syndromes. Therefore, the benefits of screening with whole-body MRI must be weighed against risks, including potential technique-related issues, false-positive imaging findings and costs. Last but not least, whole-body MRI recommendations for tumor surveillance usually do not include the age group of children ages 4-6 months and 6 years.

Summary of screening in cancer predisposition syndromes (Table 6): Whole-body MRI can reveal multifocal disease in cancer predisposition syndromes and can be complemented by dedicated regional studies with small field-of-view (FOV) imaging if indicated. The absence of ionizing radiation is an advantage in the pediatric population. Standard whole-body MRI methodology and imaging protocols for screening of many pediatric cancer predisposition syndromes are not clearly established. While modernization of MRI devices is in progress globally, the same technical level of MRI scanners, technologists' expertise and radiologists' experience in cancer predisposition syndromes interpretation cannot be guaranteed yet. There is a need for large prospective multicenter studies to establish the role, effectiveness and costs of screening with whole-body MRI in pediatric cancer predisposition syndromes. Despite the lack of standardization in the screening and evaluation of multifocal lesions in pediatric patients, whole-body MRI is certainly among the imaging methods of first choice.

Key recommendations in cancer predisposition syndromes: Whole-body MRI plays an increasing role in the surveillance of children with cancer predisposition syndromes. In cancer predisposition syndrome surveillance with whole-body MRI, knowledge of the tumor spectrum per syndrome and knowledge of the imaging characteristics of specific tumors and normal variants are mandatory to improve lesion detection and reduce false-positive findings.

\section{General technical considerations}

Given a larger field of view and a more stable magnetic field, a 1.5-tesla (T) scanner might be preferable to a 3.0-T device for whole-body MRI. The diagnostic performance of MRI depends substantially on the sequence type and scan parameters, especially the spatial resolution. The variety of technical options renders it a versatile modality, but for the same reasons it is highly operator-dependent and difficult to standardize. The often long duration of the examination directly affects patient comfort. Thus, a modular approach consisting of basic and
Table 6 Whole-body magnetic resonance imaging surveillance in cancer predisposition syndromes

\begin{tabular}{|c|c|c|c|c|c|}
\hline \multirow[t]{2}{*}{ Disease } & \multicolumn{4}{|c|}{ Body sequences } & \multirow{2}{*}{$\begin{array}{l}\text { MRI brain, } \\
\text { dedicated }\end{array}$} \\
\hline & $\begin{array}{l}\text { T2-W } \\
\text { STIR } \\
\text { coronal }\end{array}$ & $\begin{array}{l}\text { T1-W } \\
\text { TSE } \\
\text { coronal }\end{array}$ & $\begin{array}{l}\text { DWI axial } \\
\text { (coronal } \\
\text { reconstruction) }\end{array}$ & $\begin{array}{l}\text { T2-W SPAIR axial } \\
\text { (thorax, abdomen, }^{\mathrm{a}} \\
\text { breath hold or } \\
\text { respiratory } \\
\text { triggering) }\end{array}$ & \\
\hline $\begin{array}{l}\text { Li-Fraumeni } \\
\text { syndrome }\end{array}$ & \multicolumn{4}{|c|}{ Whole-body MRI every 12 months from diagnosis ${ }^{\mathrm{b}}$} & Every 6 months ${ }^{\mathrm{b}}$ \\
\hline $\begin{array}{l}\text { Congenital } \\
\text { mismatch } \\
\text { repair syndrome }\end{array}$ & \multicolumn{4}{|c|}{ Whole-body MRI every 12 months from 6 years to 8 years } & Not indicated \\
\hline $\begin{array}{l}\text { Hereditary } \\
\text { retinoblastoma }\end{array}$ & \multicolumn{4}{|c|}{ Whole-body MRI every 12 months from 8 years to 10 years } & $\begin{array}{c}\text { Every } 6 \text { months to } \\
5 \text { years of age }\end{array}$ \\
\hline $\begin{array}{l}\text { Neurofibromatosis } \\
1 \text { and } \\
\text { neurofibromato- } \\
\text { sis } 2\end{array}$ & \multicolumn{4}{|c|}{$\begin{array}{l}\text { Whole-body MRI baseline scan at 16-20 years (no need for } \\
\text { whole-body MRI surveillance unless symptomatic or } \\
\text { detected tumor); whole-body MRI baseline }\end{array}$} & Indicated \\
\hline
\end{tabular}

$D W I$ diffusion-weighted imaging, SPAIR spectrally adiabatic inversion recovery, STIR short tau inversion recovery, T1-W T1-weighted, T2-W T2-weighted, TSE turbo spin echo

${ }^{\mathrm{a}}$ Optional

${ }^{\mathrm{b}}$ Whole-body MRI and MRI brain interleaved at 6-month intervals if no general anesthetic is needed 
advanced sequences is useful $[4,13,17,18,67,68]$. An example of a whole-body MRI protocol from the pediatric radiology department at the University Hospital Tübingen is shown in Table 7. The modular concept facilitates the adjustment of the protocol to a particular indication and also improves the reproducibility and comparability of individual examinations.

In young children the company of a parent, adequate introduction to the examination, and distraction by audio or video equipment during the scan help to reduce motion artifacts and even the necessity of sedation or general anesthesia. These preparations might seem time-consuming, but their influence on image quality should not be underestimated.

In agreement with previous publications, we propose a high-resolution fat-saturated T2-weighted sequence (e.g., STIR) with slice thicknesses of 3-4 $\mathrm{mm}$ in coronal orientation as the mainstay of the basic module. To avoid limitations due to partial volume effects, additional sections in transverse orientation in the regions of the head, neck and trunk are recommended [4, 13, 17, 18, 67-69]. For reduction of motion artifacts, radial $k$-space acquisition and navigator techniques are helpful. Electrocardiography-triggered sequences might improve the depiction of small lesions in the mediastinum or in the lung. Sequences or reconstructions in sagittal orientation are necessary when focusing on spinal pathologies. Advanced modules comprise (whole-body) diffusion-weighted and T1weighted sequences before and after administration of contrast medium. After contrast injection, fat suppression is mandatory.
In addition to whole-body examinations, dedicated imaging of body regions such as orbits, hands and feet, and the evaluation of small lesions in residual or relapsing disease require optimized equipment and coil configuration $[4,13$, 17, 18, 67-69]. Particularly in the case of children who can only be examined under general anesthesia or sedation, additional imaging (e.g., local MRI) might be replaced by this comprehensive approach. This advantage often outweighs the necessary extension of the examination time [16].

In the literature, most protocol recommendations for whole-body MRI in children and adolescents give a fixed FoV, resolution matrix and slice thickness and thus a fixed voxel size. However, as is typical in pediatric MRI, these sequence parameters should be adapted to the indication as well as the various body sizes from infant to adolescent because the spatial resolution is significant for diagnostic sensitivity [70]. Especially in cases where sequences are acquired in a single (coronal) orientation, a sufficiently high resolution is crucial. Therefore, the following approach might be helpful: based on a given resolution matrix (e.g., $256 \times 256-384 \times$ 384 ), the voxel size is automatically reduced by adjusting the FOV to the child's size and by moderately adjusting the slice thickness to a smaller body size, resulting in relatively constant anatomical resolution/image information. Therefore, for morphological assessment we recommend a voxel size in children younger than 1 year of $1 \times 1 \times 3 \mathrm{~mm}^{3}$ in the coronal plane and of $0.8 \times 0.8 \times 3.0 \mathrm{~mm}^{3}$ in the transverse plane. However, the subsequent signal loss should be compensated (e.g., by increase of the in-phase oversampling or the signal

Table 7 Example of a modular concept of whole-body magnetic resonance imaging protocol

\begin{tabular}{|c|c|c|c|c|c|}
\hline Module & Region & Sequence & $\begin{array}{l}\text { Orientation/phase } \\
\text { encoding direction }\end{array}$ & $\begin{array}{l}\text { Base matrix } \\
\text { (FOV adapted to } \\
\text { child's size) }\end{array}$ & Remarks \\
\hline \multirow[t]{4}{*}{ Basic module } & Whole body & $\begin{array}{l}\text { 2-D STIR } \\
\text { TSE/FSE }\end{array}$ & Coronal/FH & 384 & \\
\hline & Head and neck & $\begin{array}{l}\text { 2-D STIR } \\
\text { TSE/FSE }\end{array}$ & Axial/AP & 384 & Head to aortic arch \\
\hline & Thorax & $\begin{array}{l}\text { 2-D T2-W } \\
\text { TSE/FSE } \\
\text { fat-saturated } \\
\text { navigator }\end{array}$ & Axial/AP & 384 & $\begin{array}{l}\text { Radial k-space sampling } \\
\text { with respiratory triggering }\end{array}$ \\
\hline & $\begin{array}{l}\text { Abdomen and } \\
\text { pelvis }\end{array}$ & $\begin{array}{l}\text { 2-D T2-W } \\
\text { TSE/FSE } \\
\text { fat-saturated } \\
\text { navigator }\end{array}$ & Axial/AP & 384 & $\begin{array}{l}\text { Radial k-space sampling } \\
\text { with respiratory triggering }\end{array}$ \\
\hline DWI & Whole body & $\begin{array}{c}2-\mathrm{D} \text { EPI (SPAIR) } \\
2 \text { b values: } 0 \text { and } \\
1,000 \mathrm{~s} / \mathrm{mm}^{2}\end{array}$ & Axial/AP & 128 & $\begin{array}{l}\text { Coronal MPR; ADC map; } \\
\text { calculation of high b } \\
\text { value } \geq 1,200 \mathrm{~s} / \mathrm{mm}^{2}\end{array}$ \\
\hline $\mathbf{C E}$ & Whole body & $\begin{array}{l}\text { 3-D T1-W GRE } \\
\text { (VIBE/Dixon) }\end{array}$ & Axial/AP & $288-320$ & If possible breath hold; coronal MPR \\
\hline
\end{tabular}

$A D C$ apparent diffusion coefficient, $A P$ anterior-to-posterior, $C E$ contrast-enhanced, $D W I$ diffusion-weighted imaging, $E P I$ echoplanar imaging, $F H$ feet-to-head, FSE fast spin echo, GRE gradient echo, MPR multiplanar reconstruction, SPAIR spectral attenuated inversion recovery, STIR short tau inversion recovery, TSE turbo spin echo, VIBE volumetric interpolated breath-hold examination 
averaging), resulting in a longer acquisition time. Therefore, an optimal compromise between tolerable signal-to-noise ratio loss and measurement time should be found. It is advantageous that the number of blocks/stations in the Z-direction and thus the total acquisition time can be reduced based on the body geometry of small children. The use of predefined protocols optimized to different body sizes facilitates adaptation in daily practice.

Acknowledgments Open Access funding provided by Projekt DEAL.

\section{Compliance with ethical standards}

\section{Conflicts of interest None}

Collaborators The Oncology Task Force of the ESPR. Collaborators in alphabetic order:

Mehmet Burak Özkan, School of Medicine, Samsun

Angela Byrne, Our Lady's Hospital for Sick Children, Dublin

Anne Thora Grammeltvedt, St. Olavs University Hospital, Trondheim

Kieran McHugh, Great Ormond Street Hospital for Children, London

Dhananjaya Kotebagilu Narayana Vamyanmane, Isha Diagnostics and Research, Bengaluru

Open Access This article is licensed under a Creative Commons Attribution 4.0 International License, which permits use, sharing, adaptation, distribution and reproduction in any medium or format, as long as you give appropriate credit to the original author(s) and the source, provide a link to the Creative Commons licence, and indicate if changes were made. The images or other third party material in this article are included in the article's Creative Commons licence, unless indicated otherwise in a credit line to the material. If material is not included in the article's Creative Commons licence and your intended use is not permitted by statutory regulation or exceeds the permitted use, you will need to obtain permission directly from the copyright holder. To view a copy of this licence, visit http://creativecommons.org/licenses/by/4.0/.

\section{References}

1. Daldrup-Link HE, Franzius C, Link TM et al (2001) Whole-body MR imaging for detection of bone metastases in children and young adults: comparison with skeletal scintigraphy and FDG PET. AJR Am J Roentgenol 177:229-236

2. Kellenberger CJ, Miller SF, Khan M et al (2004) Initial experience with FSE STIR whole-body MR imaging for staging lymphoma in children. Eur Radiol 14:1829-1841

3. Goo HW, Yang DH, Ra YS et al (2006) Whole-body MRI of Langerhans cell histiocytosis: comparison with radiography and bone scintigraphy. Pediatr Radiol 36:1019-1031

4. Schaefer JF, Schlemmer HP (2006) Total-body MR-imaging in oncology. Eur Radiol 16:2000-2015

5. Burdach S, Thiel U, Schoniger M et al (2010) Total body MRI-governed involved compartment irradiation combined with high-dose chemotherapy and stem cell rescue improves long-term survival in Ewing tumor patients with multiple primary bone metastases. Bone Marrow Transplant 45:483489
6. Kwee TC, Takahara T, Vermoolen MA et al (2010) Whole-body diffusion-weighted imaging for staging malignant lymphoma in children. Pediatr Radiol 40:1592-1602

7. Siegel MJ, Acharyya S, Hoffer FA et al (2013) Whole-body MR imaging for staging of malignant tumors in pediatric patients: results of the American College of Radiology Imaging Network 6660 trial. Radiology 266:599-609

8. Littooij AS, Kwee TC, Barber I et al (2014) Whole-body MRI for initial staging of paediatric lymphoma: prospective comparison to an FDG-PET/CT-based reference standard. Eur Radiol 24:1153-1165

9. Littooij AS, Kwee TC, de Keizer B et al (2015) Whole-body MRIDWI for assessment of residual disease after completion of therapy in lymphoma: a prospective multicenter study. J Magn Reson Imaging 42:1646-1655

10. Damasio MB, Magnaguagno F, Stagnaro G (2016) Whole-body MRI: non-oncological applications in paediatrics. Radiol Med 121:454-461

11. Ballinger ML, Best A, Mai PL et al (2017) Baseline surveillance in Li-Fraumeni syndrome using whole-body magnetic resonance imaging: a meta-analysis. JAMA Oncol 3:1634-1639

12. Albano D, Patti C, Matranga D et al (2018) Whole-body diffusionweighted MR and FDG-PET/CT in Hodgkin lymphoma: predictive role before treatment and early assessment after two courses of ABVD. Eur J Radiol 103:90-98

13. Schaefer JF, Berthold LD, Hahn G et al (2019) Whole-body MRI in children and adolescents - S1 guideline. Rofo 191:618-625

14. Kellenberger CJ, Epelman M, Miller SF, Babyn PS (2004) Fast STIR whole-body MR imaging in children. Radiographics 24 : $1317-1330$

15. Mentzel HJ, Kentouche K, Sauner D et al (2004) Comparison of whole-body STIR-MRI and $99 \mathrm{mTc}$-methylene-diphosphonate scintigraphy in children with suspected multifocal bone lesions. Eur Radiol 14:2297-2302

16. Goo HW (2011) Regional and whole-body imaging in pediatric oncology. Pediatr Radiol 41:S186-S194

17. Greer MC (2018) Whole-body magnetic resonance imaging: techniques and non-oncologic indications. Pediatr Radiol 48:13481363

18. Ley S, Ley-Zaporozhan J, Schenk JP (2009) Whole-body MRI in the pediatric patient. Eur J Radiol 70:442-451

19. Ishiguchi H, Ito $\mathrm{S}$, Kato $\mathrm{K}$ et al (2018) Diagnostic performance of (18)F-FDG PET/CT and whole-body diffusion-weighted imaging with background body suppression (DWIBS) in detection of lymph node and bone metastases from pediatric neuroblastoma. Ann Nucl Med 32:348-362

20. Klenk C, Gawande R, Uslu L et al (2014) Ionising radiation-free whole-body MRI versus (18)F-fluorodeoxyglucose PET/CT scans for children and young adults with cancer: a prospective, nonrandomised, single-centre study. Lancet Oncol 15:275-285

21. Punwani S, Prakash V, Bainbridge A et al (2010) Quantitative diffusion weighted MRI: a functional biomarker of nodal disease in Hodgkin lymphoma? Cancer Biomark 7:249-259

22. Wu X, Pertovaara H, Dastidar P et al (2013) ADC measurements in diffuse large b-cell lymphoma and follicular lymphoma: a DWI and cellularity study. Eur J Radiol 82:e158-e164

23. Punwani S, Cheung KK, Skipper N et al (2013) Dynamic contrastenhanced MRI improves accuracy for detecting focal splenic involvement in children and adolescents with Hodgkin disease. Pediatr Radiol 43:941-949

24. Raissaki M, Demetriou S, Spanakis K et al (2017) Multifocal bone and bone marrow lesions in children - MRI findings. Pediatr Radiol 47:342-360 
25. Steinborn M, Wortler K, Nathrath M et al (2008) Whole-body MRI in children with Langerhans cell histiocytosis for the evaluation of the skeletal system. Rofo 180:646-653

26. Smets AM, Deurloo EE, Slager TJE et al (2018) Whole-body magnetic resonance imaging for detection of skeletal metastases in children and young people with primary solid tumors - systematic review. Pediatr Radiol 48:241-252

27. Kumar J, Seith A, Kumar A et al (2008) Whole-body MR imaging with the use of parallel imaging for detection of skeletal metastases in pediatric patients with small-cell neoplasms: comparison with skeletal scintigraphy and FDG PET/CT. Pediatr Radiol 38:953-962

28. Krohmer S, Sorge I, Krausse A et al (2010) Whole-body MRI for primary evaluation of malignant disease in children. Eur J Radiol 74:256-261

29. Punwani S, Taylor SA, Bainbridge A et al (2010) Pediatric and adolescent lymphoma: comparison of whole-body STIR halfFourier RARE MR imaging with an enhanced PET/CT reference for initial staging. Radiology 255:182-190

30. Regacini R, Puchnick A, Luisi FAV, Lederman HM (2018) Can diffusion-weighted whole-body MRI replace contrast-enhanced CT for initial staging of Hodgkin lymphoma in children and adolescents? Pediatr Radiol 48:638-647

31. Anupindi SA, Bedoya MA, Lindell RB et al (2015) Diagnostic performance of whole-body MRI as a tool for cancer screening in children with genetic cancer-predisposing conditions. AJR Am J Roentgenol 205:400-408

32. Benusiglio PR, Brugieres L, Caron O (2016) Whole-body MRI screening in children with $\mathrm{Li}$-Fraumeni and other cancer predisposition syndromes. AJR Am J Roentgenol 206:W52

33. Bojadzieva J, Amini B, Day SF et al (2017) Whole body magnetic resonance imaging (WB-MRI) and brain MRI baseline surveillance in TP53 germline mutation carriers: experience from the LiFraumeni syndrome education and early detection (LEAD) clinic. Familial Cancer 17:287-294

34. O'Neill AF, Voss SD, Jagannathan JP et al (2018) Screening with whole-body magnetic resonance imaging in pediatric subjects with Li-Fraumeni syndrome: a single institution pilot study. Pediatr Blood Cancer 65

35. Mautner VF, Asuagbor FA, Dombi E et al (2008) Assessment of benign tumor burden by whole-body MRI in patients with Neurofibromatosis 1. Neuro Oncol 10:593-598

36. Nguyen R, Jett K, Harris GJ et al (2014) Benign whole body tumor volume is a risk factor for malignant peripheral nerve sheath tumors in neurofibromatosis type 1. J Neurooncol 116:307-313

37. Evans DGR, Salvador H, Chang VY (2017) Cancer and central nervous system tumor surveillance in pediatric Neurofibromatosis 1. Clin Cancer Res 23:e46-e53

38. Friedman DN, Lis E, Sklar CA et al (2014) Whole-body magnetic resonance imaging (WB-MRI) as surveillance for subsequent malignancies in survivors of hereditary retinoblastoma: a pilot study. Pediatr Blood Cancer 61:1440-1444

39. Siegel RL, Miller KD, Jemal A (2015) Cancer statistics, 2015. CA Cancer J Clin 65:5-29

40. Cheson BD, Fisher RI, Barrington SF et al (2014) Recommendations for initial evaluation, staging, and response assessment of Hodgkin and non-Hodgkin lymphoma: the Lugano classification. J Clin Oncol 32:3059-3068

41. Rosolen A, Perkins SL, Pinkerton CR et al (2015) Revised international pediatric non-Hodgkin lymphoma staging system. J Clin Oncol 33:2112-2118

42. Latifoltojar A, Punwani S, Lopes A (2019) Whole-body MRI for staging and interim response monitoring in paediatric and adolescent
Hodgkin's lymphoma: a comparison with multi-modality reference standard including (18)F-FDG-PET-CT. Eur Radiol 29:202-212

43. Mayerhoefer ME, Karanikas G, Kletter K (2015) Evaluation of diffusion-weighted magnetic resonance imaging for follow-up and treatment response assessment of lymphoma: results of an $18 \mathrm{~F}$ FDG-PET/CT-controlled prospective study in 64 patients. Clin Cancer Res 21:2506-2513

44. Lin C, Itti E, Luciani A et al (2011) Whole-body diffusion-weighted imaging with apparent diffusion coefficient mapping for treatment response assessment in patients with diffuse large B-cell lymphoma: pilot study. Investig Radiol 46:341-349

45. Meyer JS, Siegel MJ, Farooqui SO et al (2005) Which MRI sequence of the spine best reveals bone-marrow metastases of neuroblastoma? Pediatr Radiol 35:778-785

46. Papaioannou G, McHugh K (2005) Neuroblastoma in childhood: review and radiological findings. Cancer Imaging 5:116-127

47. Monclair T, Brodeur GM, Ambros PF et al (2009) The International Neuroblastoma Risk Group (INRG) staging system: an INRG task force report. J Clin Oncol 27:298-303

48. Pfluger T, Schmied C, Porn U et al (2003) Integrated imaging using MRI and 123I metaiodobenzylguanidine scintigraphy to improve sensitivity and specificity in the diagnosis of pediatric neuroblastoma. AJR Am J Roentgenol 181:1115-1124

49. Kembhavi SA, Rangarajan V, Shah S et al (2014) Prospective observational study on diagnostic accuracy of whole-body MRI in solid small round cell tumours. Clin Radiol 69:900-908

50. Peschmann AL, Beer M, Ammann B et al (2019) Quantitative DWI predicts event-free survival in children with neuroblastic tumours: preliminary findings from a retrospective cohort study. Eur Radiol Exp 3:6

51. Casali PG, Abecassis N, Aro HT et al (2018) Soft tissue and visceral sarcomas: ESMO-EURACAN clinical practice guidelines for diagnosis, treatment and follow-up. Ann Oncol 29:iv268-iv269

52. Casali PG, Bielack S, Abecassis N et al (2018) Bone sarcomas: ESMO-PaedCan-EURACAN clinical practice guidelines for diagnosis, treatment and follow-up. Ann Oncol 29:iv79-iv95

53. Ben Arush M, Minard-Colin V, Mosseri V et al (2015) Does aggressive local treatment have an impact on survival in children with metastatic rhabdomyosarcoma? Eur J Cancer 51:193-201

54. Federico SM, Spunt SL, Krasin MJ et al (2013) Comparison of PET-CT and conventional imaging in staging pediatric rhabdomyosarcoma. Pediatr Blood Cancer 60:1128-1134

55. Scheer M, Dantonello T, Brossart P et al (2018) Importance of whole-body imaging with complete coverage of hands and feet in alveolar rhabdomyosarcoma staging. Pediatr Radiol 48:648-657

56. Goo HW, Choi SH, Ghim T et al (2005) Whole-body MRI of paediatric malignant tumours: comparison with conventional oncological imaging methods. Pediatr Radiol 35:766-773

57. Norman G, Fayter D, Lewis-Light K et al (2015) Mind the gap: extent of use of diffusion-weighted MRI in children with rhabdomyosarcoma. Pediatr Radiol 45:778-781

58. Kubo T, Furuta T, Johan MP et al (2017) Value of diffusionweighted imaging for evaluating chemotherapy response in osteosarcoma: a meta-analysis. Mol Clin Oncol 7:88-92

59. Morimoto A (2017) Langerhans cell histiocytosis. In: Ishii E (ed) Hematological disorders in children: pathogenesis and treatment. Springer, Singapore

60. Kim JR, Yoon HM, Jung AY et al (2019) Comparison of wholebody MRI, bone scan, and radiographic skeletal survey for lesion detection and risk stratification of Langerhans cell histiocytosis. Sci Rep 9:317

61. Laffan EE, O'Connor R, Ryan SP, Donoghue VB (2004) Wholebody magnetic resonance imaging: a useful additional sequence in paediatric imaging. Pediatr Radiol 34:472-480 
62. Mueller WP, Melzer HI, Schmid I et al (2013) The diagnostic value of $18 \mathrm{~F}-\mathrm{FDG}$ PET and MRI in paediatric histiocytosis. Eur J Nucl Med Mol Imaging 40:356-363

63. Robson ME, Bradbury AR, Arun B et al (2015) American Society of Clinical Oncology policy statement update: genetic and genomic testing for cancer susceptibility. J Clin Oncol 33:3660-3667

64. Greer MC (2018) Imaging of cancer predisposition syndromes. Pediatr Radiol 48:1364-1375

65. Villani A, Shore A, Wasserman JD et al (2016) Biochemical and imaging surveillance in germline TP53 mutation carriers with liFraumeni syndrome: 11 year follow-up of a prospective observational study. Lancet Oncol 17:1295-1305

66. Kamihara J, Bourdeaut F, Foulkes WD et al (2017) Retinoblastoma and neuroblastoma predisposition and surveillance. Clin Cancer Res 23:e98-e106
67. Darge K, Jaramillo D, Siegel MJ (2008) Whole-body MRI in children: current status and future applications. Eur J Radiol 68:289-298

68. Schaefer JF, Kramer U (2011) Whole-body MRI in children and juveniles. Rofo 183:24-36

69. Eutsler EP, Khanna G (2016) Whole-body magnetic resonance imaging in children: technique and clinical applications. Pediatr Radiol 46:858-872

70. Olsen OE (2008) Practical body MRI - a paediatric perspective. Eur J Radiol 68:299-308

Publisher's note Springer Nature remains neutral with regard to jurisdictional claims in published maps and institutional affiliations. 